\title{
Fatores para o desenvolvimento de doenças cardíacas em bebês prematuros
}

\author{
Factors for the development of heart disease in premature babies \\ Factores para el desarrollo de enfermedades del corazón en bebés prematuros
}

Recebido: 07/06/2021 | Revisado: 13/06/2021 | Aceito: 15/06/2021 | Publicado: 01/07/2021

\author{
Mariely Ravenna Coelho Galvão \\ ORCID: https://orcid.org/0000-0003-3347-9305 \\ Centro Universitário Unifacid Wyden, Brasil \\ E-mail: marielyravena@gmail.com \\ Alice Lima Rosa Mendes \\ ORCID: https://orcid.org/0000-0002-1960-9647 \\ Universidade de Brasília, Brasil \\ E-mail: alice_lima_@hotmail.com \\ Suely Moura Melo \\ ORCID: https://orcid.org/0000-0001-9996-0850 \\ Centro Universitário Unifacid Wyden, Brasil \\ E-mail: suelymouramelo@yahoo.com.br
}

\begin{abstract}
Resumo
As doenças cardiovasculares (DCV) constituem um dos principais problemas de saúde pública dos tempos atuais. Onde, segundo dados da Organização Mundial de Saúde (2017), cerca de 27\% dos registros de mortalidade no mundo foram decorrentes de DCV, enquanto no Brasil, elas foram responsáveis por $31 \%$ das mortes na população em geral. Apesar dos avanços na obstetrícia e dos avanços nos métodos de detecção precoce, os neonatos decorrentes de partos prematuros apresentam uma probabilidade duas vezes maior de apresentarem anormalidades cardíacas. Diante disso, o presente estudo pretende analisar quais os fatores para o aparecimento de DVC em bebês prematuros, identificando quais as principais causaspara a ocorrência de doenças cardíacas nos mesmos, quais cardiopatias são mais frequentes de encontrar, suas manifestações clínicas a fim de realizar o diagnóstico adequado e os procedimentos utilizados no tratamento, apresentando uma visão voltada à Biomedicina. Para tanto, realizou-se uma revisão de literatura do tipo narrativa, por meio das bases de dados do Google Acadêmico, Biblioteca Virtual em Saúde (BVS), Pub Med, Scientific Eletronic Library OnLine (SciELO). Os estudos encontrados apresentaram os fatores que podem relacionar a apresentação dedoenças cardíacas em bebês prematuros, como a idade gestacional, fatores genéticos, estilo de vida materno entre outros. Porém, apesar dos resultados encontrados ainda existem poucos arquivos a respeito do tema, fazendo-se necessário mais estudos, pois é um tema bastante relevante e pode contribuirsignificativamente para a área da saúde pública.
\end{abstract}

Palavras-chave: Doenças cardíacas; Cardiopatias congênitas; Bebês prematuros.

\begin{abstract}
Cardiovascular diseases (CVD) are one of the main public health problems of today. Where, according to data from the World Health Organization (2017), about $27 \%$ of mortality records in the world were due to CVD, while in Brazil, they accounted for $31 \%$ of deaths in the general population. Despite advances in obstetrics and advances in early detection methods, neonates resulting from premature births are twice as likely to present cardiac abnormalities. Therefore, the present study aims to analyze the factors for the onset of CVD in premature babies, identifying the main causes for the occurrence of heart diseases in them, which heart diseases are more frequent to find, their clinical manifestations in order to perform the appropriate diagnosis and the procedures used in the treatment, presenting a vision focused on Biomedicine. To this end, a literature review of the narrative type was carried out through the databases of Google Scholar, Virtual Health Library (VHL), Pub Med, Scientific Electronic Library OnLine (SciELO). The studies found presented factors that can relate the presentation of heart diseases in premature babies, such as gestational age, genetic factors, maternal lifestyle, among others. However, despite the results found there are still few files on the subject, making further studies necessary, as it is a very relevant topic and can contribute significantly to the area of public health.
\end{abstract}

Keywords: Heart disease; Congenital heart disease; Premature babies.

\section{Resumen}

Las enfermedades cardiovasculares (ECV) son uno de los principales problemas de salud pública de la actualidad. Donde, según datos de la Organización Mundial de la Salud (2017), alrededor del 27\% de los registros de mortalidad en el mundo se debieron a ECV, mientras que en Brasil, representaron el 31\% de las muertes en la población general. 
A pesar de los avances en obstetricia y los avances en los métodos de detección temprana, los neonatos resultantes de nacimientos prematuros tienen el doble de probabilidades de presentar anomalías cardíacas. Por lo tanto, el presente estudio tiene como objetivo analizar los factores para la aparición de ECV en bebés prematuros, identificando las principales causas para la aparición de enfermedades cardíacas en ellos, que las cardiopatías son más frecuentes de encontrar, sus manifestaciones clínicas con el fin de realizar el diagnóstico adecuado y los procedimientos utilizados en el tratamiento, presentando una visión centrada en la Biomedicina. Para ello, se realizó una revisión bibliográfica del tipo narrativo a través de las bases de datos de Google Scholar, Virtual Health Library (BVS), Pub Med, Scientific Electronic Library OnLine (SciELO). Los estudios encontraron que presentaban factores que pueden relacionar la presentación de enfermedades cardíacas en bebés prematuros, como la edad gestacional, factores genéticos, estilo de vida materno, entre otros. Sin embargo, a pesar de los resultados encontrados todavía hay pocos archivos sobre el tema, por lo que es necesario realizar más estudios, ya que es un tema muy relevante y puede contribuir significativamente al área de la salud pública.

Palabras clave: Enfermedad cardíaca; Cardiopatía congénita; Bebés prematuros.

\section{Introdução}

As doenças cardiovasculares (DCV) integram um dos principais problemas de saúde pública dos tempos atuais, o que pode desencadear um aumento significativo de anos perdidos da vida produtiva e mortalidade precoce Higino et al., (2015). Segundo dados da Organização Mundial de Saúde (2017), cerca de 27\% dos registros de mortalidade no mundo foram decorrentes de DCV, enquanto no Brasil, elas foram responsáveis por $31 \%$ das mortes na população em geral.

Nesse sentido, apesar de todos os avanços na obstetrícia e das melhorias nos cuidados no período do pré-natal em gestantes, os neonatos decorrentes de partos prematuros apresentam uma probabilidade duas vezes maior para apresentarem anormalidades cardíacas. Com os avanços no diagnóstico em exames de rotina na gestação é possível rastrear doenças cardíacas congênitas (DCC) e a partir daí as intervenções fetais vão ser facilitadas e irão se expandir, fazendo com que os bebês com DCC sejam uma população crescente em ascensão Axelrod; Chock; Reddy, (2016).

Quando as cardiopatias são detectadas precocemente, a comunicação à família é muito importante, pois o planejamento de medidas terapêuticas viáveis deverá ser adotado. Algumas cardiopatias congênitas e síndromes das quais fazem parte esse primeiro diagnóstico pode ser incompatível com a vida extrauterina, onde é um fator que deve ser levado em conta nos cuidados imediatos aos afetados por cardiopatias Santos; Menezes\& Sousa, (2013).

Nessa perspectiva, diante dos agravos das doenças cardíacas, percebe-se a necessidade de se avaliar os fatores para o aparecimento de doenças cardíacas em bebês prematuros. Visto que, é um assunto pouco abordado.

Então, o objetivo geral dessa pesquisa é o de identificar as principais causas para ocorrência de doenças cardíacas em bebês prematuros. Para tanto, foram delimitados os seguintes objetivos específicos: Identificar as cardiopatias congênitas; especificar as manifestações clínicas no diagnóstico das Doenças Cardíacas em bebês prematuros e identificar procedimentos utilizados em prematuros com doenças cardíacas.

\section{Metodologia}

Realizou-se uma pesquisa por meio de uma revisão bibliográfica do tipo narrativa nas bases de dados do Google Acadêmico, Biblioteca Virtual em Saúde (BVS), Pub Med, Scientific Eletronic Library Online (SciELO). Os critérios de inclusão foram artigos publicados entre os anos de 2009 à 2020 em língua portuguesa e inglesa.

Como critério de inclusão, foram utilizados os trabalhos publicados em inglês, português e espanhol disponíveis na integra, na modalidade de artigo científico (original ou revisão) ou documento normativo realizado por pesquisadores da área e que apresentasse contextualização referente a doenças cardíacas, cardiopatias congênitas e bebês. Quanto aos critérios de exclusão, levou-se em consideração aqueles que, apesar de apresentar os descritores selecionados, não abordavam diretamente à temática proposta. 


\section{Cardiopatias Congênitas}

As cardiopatias congênitas são as más formações na estrutura do coração, elas já são existentes desde o momento do nascimento. São os defeitos congênitos que afetam aproximadamente 8 entre 1.000 nascidos vivos Dadvand et al., (2009) \& Moons et al., (2009). E segundo a Comissão Nacional de Incorporação de Tecnologias do SUS (CONITE), complicações decorrentes desse quadro de cardiopatia correspondem a cerca de $10 \%$ dos óbitos infantis. Porém, algumas doenças coronarianas congênitas podem ser assintomáticas, podendo vir a ser diagnosticadas apenas na vida adulta.

Alguns fatores genéticos e ambientais são apontados como fatores para o desenvolvimento de cardiopatias congênitas, como os de herança materna: mutações, diabetes mellitus, alcoolismo, nutrição inadequada, idade superior a 40 anos, exposição a raio $\mathrm{X}$ e rubéola, uso de anfetaminas, distúrbios metabólicos de fenilcetonúria, ingestão de anticonvulsivantes, estrogênio, progesterona, lítio, varfarina ou isotretinoína. Porém, as causas exatas ainda não foram comprovadas Aguiar et al., (2018).

O que se sabe, é que a maioria dos bebês com cardiopatias congênitas (CC) nascem antes de 39-40 semanas de gestação, o que vai fazer com eles não tenham a maturidade fisiológica suficiente dos órgãos, e corram substancialmente mais riscos de imaturidade dos órgãos em geral Costello et al., (2010); Krishnamurthy; Ratner \& Bacha, (2013). Isso porque, nas Cardiopatias congênitas há a malformação no coração e vasos calibrosos, onde podem manifestar defeitos cardíacos individuais ou juntamente com aspectos patogênicos e fisiopatológicos Santos; Menezes \& Sousa, (2013).

Os bebês que nascem aparentemente sadios, recebem alta hospitalar entre as 36 e 48 horas de vida, onde geralmente as manifestações clínicas das cardiopatias críticas podem não ter ocorrido ainda. A maior preocupação, é o fato que $30 \%$ dos recém-nascidos recebem alta hospitalar sem o diagnóstico, o que pode fazer com eles tenham complicações mais graves Sociedade brasileira de pediatria [SBP], (2011).

Existe uma grande variedade de defeitos cardíacos, devido as várias combinações patológicas das diferentes estruturas cardíacas (átrios, ventrículos, paredes dos vasos, grandes artérias, veias e válvulas). As cardiopatias podem ser caracterizadas de acordo com os critérios patogênicos (classificação patogênica), anatômicas (classificação anatômica) ou funcionais (abordagem clínica). Sendo a última mais utilizada, pois o aspecto funcional da cardiopatia é o que vai determinar o manejo terapêutico adequado ao nascimento Mangili; Garzoli \& Sadou, (2018).

As cardiopatias congênitas integram o grupo mais comum de anomalias inatas. Onde, $25 \%$ delas são críticas e vão necessitar de intervenção cirúrgica ou cateterismo durante o primeiro ano de vida Morais \& Mimoso, (2013). Elas podem ser classificadas em cianóticas e acianóticas, e subdividas, onde são descritas como: malformações que provocam aumento do fluxo sanguíneo pulmonar; as que causam diminuição do fluxo sanguíneo pulmonar; as que provocam obstrução do fluxo sanguíneo e as anomalias associadas à cianose Aguiar et al., (2018).

Nas denominadas cianóticas vai acontecer a interferência no fluxo sanguíneo através dos pulmões, onde vai causar a diminuição de oxigênio na circulação, podendo vir a causar uma cianose generalizada. E as acianóticas, o sangue que é rico em oxigênio vai ser encaminhado para a circulação sistêmica por meio de shunting (furos ou passagens), que vão ocorrer do lado esquerdo do coração para o lado direito Queiroz \& Lucena, (2020)

As cardiopatias sem nenhum tipo (Figura 1) e as cardiopatias que causam excesso de fluxo sanguíneo para o pulmão, são: a comunicação interventricular (Figura 2), que é caracterizada como o tipo de cardiopatia congênita que se caracteriza pela abertura da parede que separa o ventrículo esquerdo do ventrículo direito. A persistência do canal arterial (Figura 3), que é a alteração que se dá quando o vaso sanguíneo que liga a aorta à artéria pulmonar, que é chamado canal arterial, não fecha dentro do prazo de 48 horas após o nascimento. E a comunicação interatrial (Figura 4), que é denominada como o defeito congênito de fechamento do septo interatrial, que divide o coração entre os lados direito e esquerdo, equivale a 35\% de todos os defeitos congênitos Lopes \& Mesquita, (2014). 
Research, Society and Development, v. 10, n. 7, e50710716917, 2021

(CC BY 4.0) | ISSN 2525-3409 | DOI: http://dx.doi.org/10.33448/rsd-v10i7.16917

Figura 1 - Coração sem nenhum tipo de cardiopatia congênita.

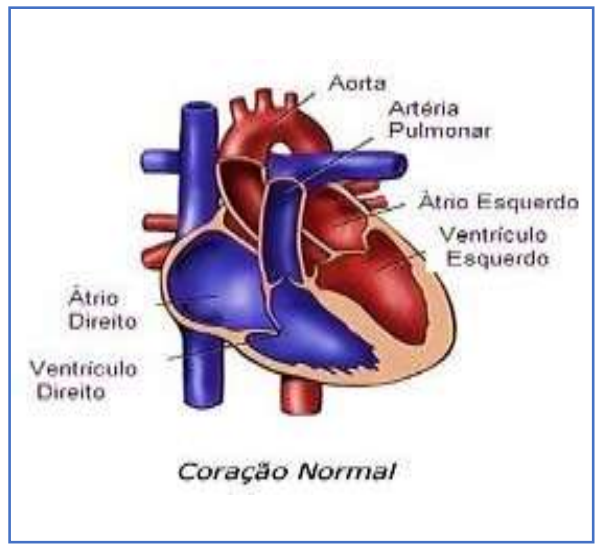

Fonte: Adaptado de figura do site Prematuridade.com (www.prematuridade.com.br).

Figura 2 - Comunicação interventricular, evidenciação da abertura da parede que separa o ventrículo esquerdo do ventrículo direito.

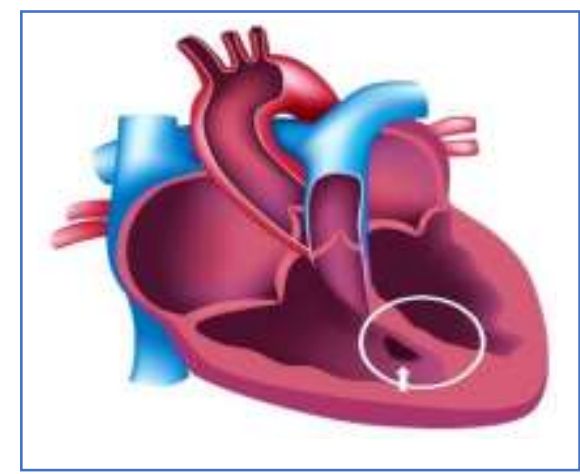

Fonte: Adaptado do site do Hospital infantil Sabará (www.hospitalinfantilsabara.org.br).

Figura 3 - Persistência do canal arterial, evidenciação da abertura do vaso sanguíneo que liga a aorta a artéria pulmonar.

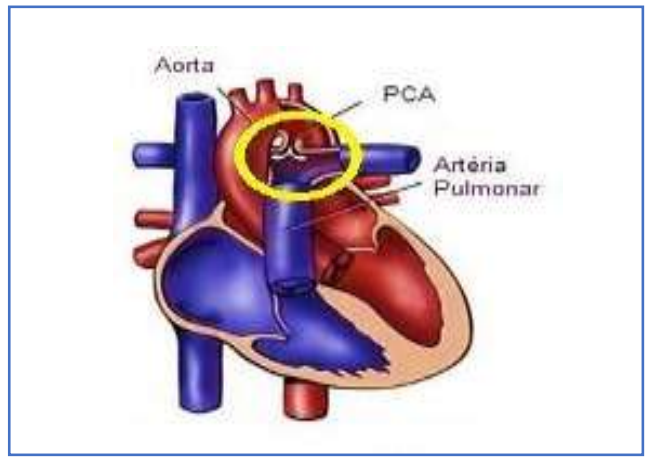

Fonte: Adaptado de figura do site Prematuridade.com (www.prematuridade.com.br). 
Figura 4 - Comunicação interatrial, identificação do fechamento interatrial, que liga o coração entre os lados direito e esquerdo.

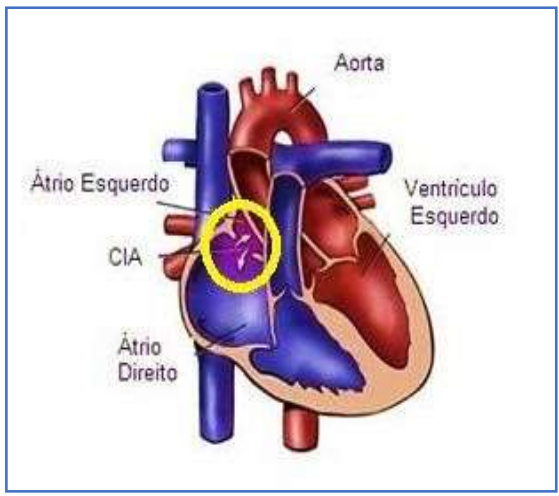

Fonte: Adaptado de figura do site Dr Bruno Rocha cirurgia cardiovascular (www.brunorocha.com.br).

As que causam diminuição do fluxo sanguíneo para o pulmão, são: Estenose pulmonar e tetralogia de Fallot. A estenose pulmonar é quando há um estreitamento da válvula pulmonar fazendo com que haja uma obstrução do sangue na passagem do fluxo sanguíneo do ventrículo direito para a artéria pulmonar (Figura 5). Já a tetralogia de Fallot (Figura 6), é caracterizada por um conjunto de alterações no coração, nos quais foram descritas por Étienne Louis Arthut Fallot no ano de 1888, e as alterações anatômicas são: comunicação interventricular, estenose da artéria pulmonar, dextroposição da artéria aorta e hipertrofia do ventrículo direito, ocorre em cada 3 de 10.000 nascidos vivos Bailliard \& Anderson, (2009).

Figura 5 - Estenose pulmonar, evidenciação do estreitamento da válvula pulmonar.

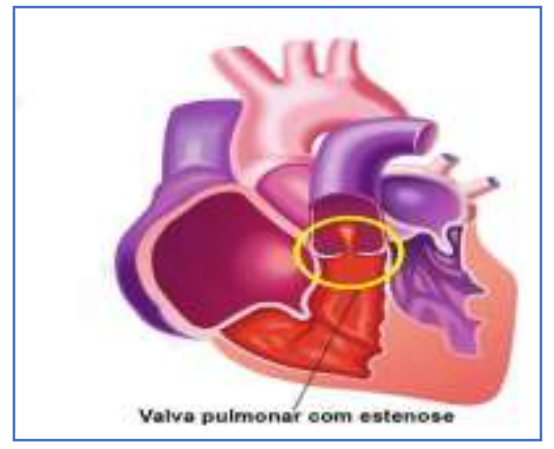

Fonte: Adaptado de figura do site Coluna Cleber Toledo (www.clebertoledo.com.br).

Figura 6 - Tetralogia de Fallot, conjunto de alterações no coração.

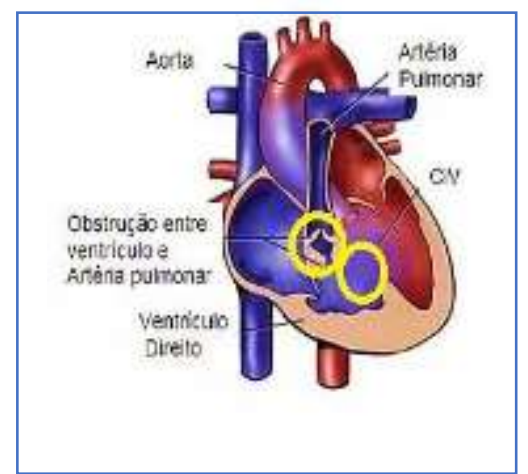

Fonte: Adaptado de figura do Blog pediatria (pediatriaufcspa.wixsite.com). 
Já as denominadas cardiopatias que causam obstrução da passagem de fluxo sanguíneos, são: Coarctação da aorta, estenose valvar aórtica e estenose sub valvar aórtica. A coarctação da aorta (Figura 7) é uma alteração, um estreitamento na região ístimica da aorta, entre a artéria subclávia esquerda e o ducto arteriosus, ela é uma das cardiopatias responsável por cerca de 5 a $8 \%$ das cardiopatias em geral Carvalho et al., (2012). A estenose valvar aórtica (Figura 8), é um estreitamento que vai dificultar a abertura a abertura da válvula aórtica para a passagem do sangue. E a estenoso subvalvar da aorta é uma obstrução do fluxo sanguíneo do ventrículo esquerdo Cortellazzi, (2017).

Figura 7 - Coarctação da aorta, evidenciação do estreitamento na abertura da válvula aórtica.

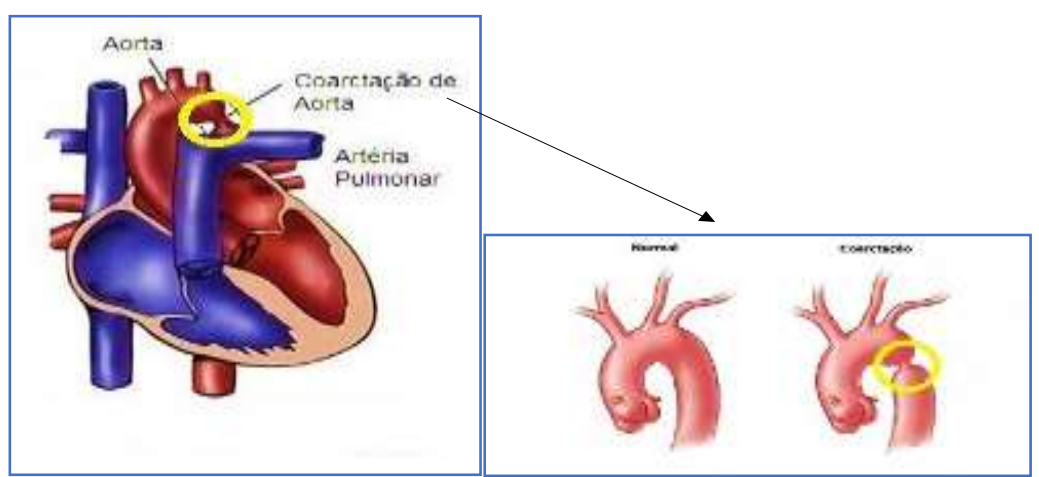

Fonte: Adapatado da figura do site Dr Bruno Rocha cirúrgia cardiovascular (www.brunorocha.com.br); Blog Coração moderno (www.coracaomoderno.com.br).

Figura 8 - Estenose valvar aórtica, identificando o defeito na válvula aórtica.

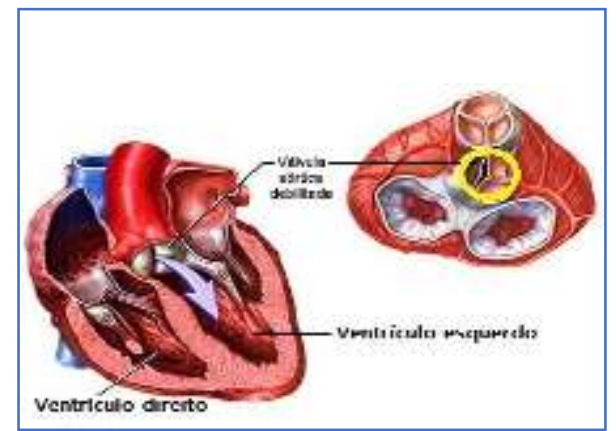

Fonte: Adaptado da figura do site Hci med (www.hci.med.br).

\section{Manifestações Clínicas para o Diagnóstico de Doenças Cardíacas em Bebês Prematuros}

O sistema cardiovascular é formado por células de origem mesodérmica. É o primeiro sistema que se desenvolve no embrião, e o primeiro que começa a funcionar. O sistema vascular e o coração primitivo se formam durante a $3^{\circ}$ semana de desenvolvimento e já na $4^{\circ}$ semana o coração começa a bater Valdes, (2013).

Depois da descoberta da gravidez, o início do pré-natal é de suma importância. O principal objetivo do pré-natal é acolher as gestantes desde o início da gravidez, objetivando concluir a gestação e com a expectativa do nascimento de uma criança saudável, garantindo o bem estar materno e do neonato. No pré-natal deve- se ter no mínimo seis consultas, uma no primeiro trimestre, duas no segundo e três no último trimestre Santos; Menezes \& Sousa, (2013).

Os passos do pré-natal são exemplificados na (Figura 9), onde na primeira consulta da gestante será realizada a anamnese, na qual serão identificados os aspectos socioepidemiológicos, antecedentes familiares, antecedentes ginecológicos e obstétricos, seus antecedentes pessoais gerais, e como está a atual situação da gravidez. O segundo passo é a história clínica, 
onde entra a identificação da paciente, dados socioeconômicos, antecedentes familiares, antecedentes ginecológicos, sexualidade, antecedentes obstétricos e perguntas a respeito da gestação atual Ministério da saúde (2012).

Figura 9 - Pré Natal.

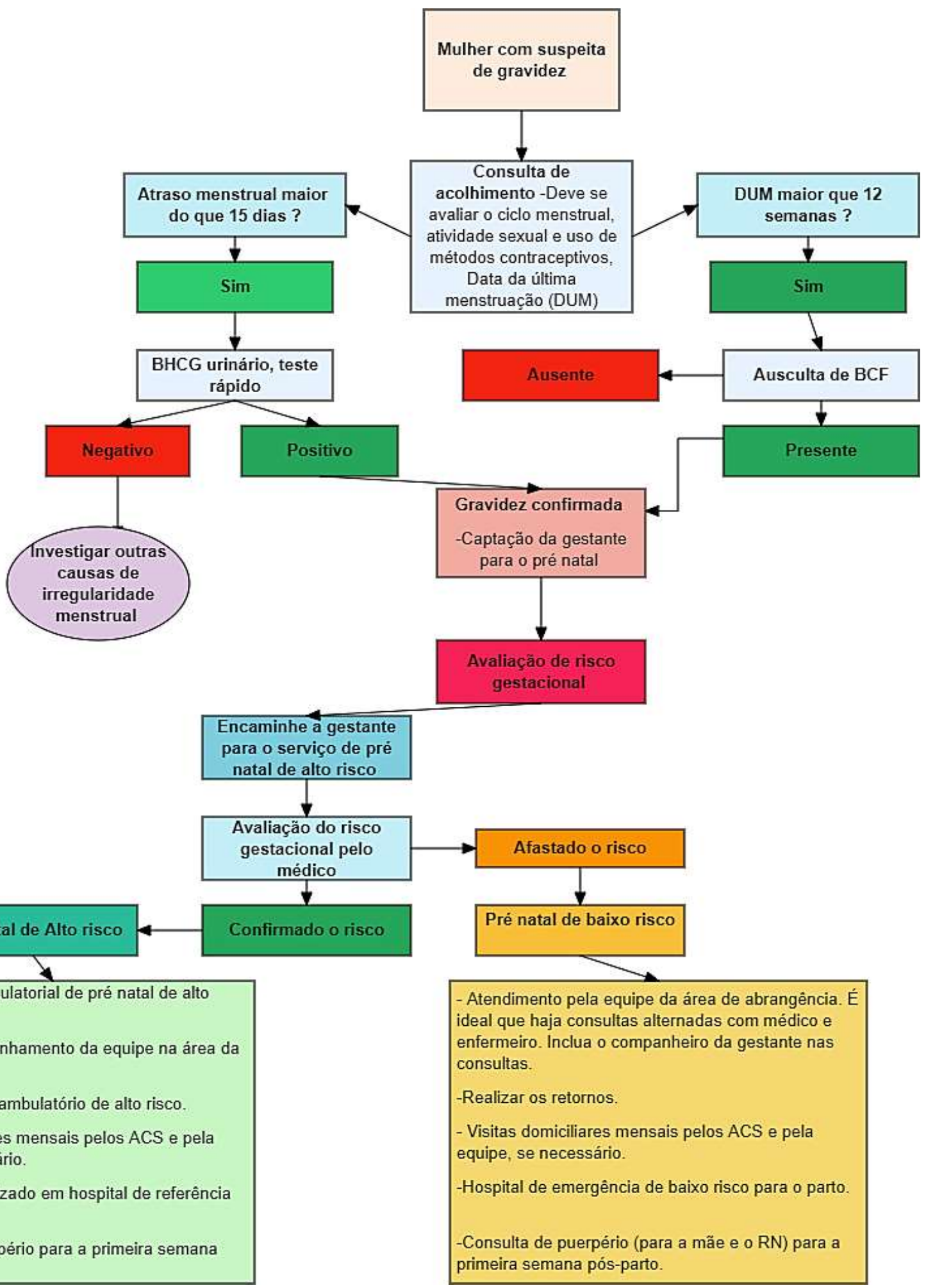

Fonte: Fluxograma Adaptado de Ministério da Saúde (2012). 
Logo depois, vem a parte do exame físico geral, onde é feita a determinação do peso, altura, cálculo do Índice de Massa Corpórea (IMC), a avaliação do estado nutricional e ganho de peso gestacional, aferição da pressão arterial, a inspeção da pele e mucosas, os sinais vitais (pulso, frequência cardíaca, frequência respiratória, temperatura axilar), palpação da tireoide, ausculta cardiopulmonar, exame do abdômen, exame dos membros inferiores, pesquisa de edemas. E os exames físicos específicos (gineco-obstétricos), onde é feita a palpação obstétrica, medida e avaliação da altura uterina, ausculta dos batimentos cardiofetais, o registro dos movimentos fetais, o teste do estímulo sonoro, o exame clínico das mamas e o exame ginecológico Ministério da saúde, (2012).

Além disso, são solicitados alguns exames complementares, os laboratoriais, entre eles: Hemograma, tipagem sanguínea e fator Rh, coombs indireto (se o Rh for negativo), glicemia em jejum, teste rápido de sífilis e/ou VDRL/RPR, teste rápido para HIV, Toxoplasmose IgM e IgG, sorologia para hepatite B, exame de urina e urocultura, ultrassonografia obstétrica (de acordo a idade gestacional), citopatológico do colo do útero (caso seja necessário), exame da secreção vaginal (se houver indicação clínica), parasitológico de fezes (caso haja indicação clínica) e eletroforese de hemoglobina, no caso de gestante negra, com antecedentes familiares que apresentem anemia falciforme ou histórico de anemia crônica Balsells et al., (2018).

Depois da avaliação de todos os exames pelo médico obstetra, deve ser realizada a classificação de risco gestacional, onde cada gestante ser encaminhada ao serviço de saúde para receber o cuidado específico necessário. No caso do SUS, as que são classificadas com fatores de risco que permitem a realização de pré-natal pela equipe de atenção básica são os casos de gestantes com idade menor que 15 anos e maior de 35 anos, aquelas que apresentam maior ocupação, fazem esforço físico excessivo, tem uma carga horária extensa, estresse e exposição a agentes químicos, físicos, biológicos, estão em situação familiar insegura e não aceitação da gravidez, situação conjugal insegura, baixa escolaridade, altura menor que 1,45, IMC que evidencie baixo peso, e apresente alguns fatores relacionados a gestação anteriores, como por exemplo síndromes hemorrágicas ou hipotensivas ou mesmo parto prematuro Secretaria de estado da saúde do Rio Grande do Sul, (2018).

Já os fatores de risco que podem indicar encaminhamento ao pré-natal de alto risco, vão fazer com que as gestantes tenham maior probabilidade de óbito materno/fetal e outras intercorrências. Essa classificação corresponde a $10 \%$ das gestações, e esses fatores são: cardiopatias, pneumonias graves, nefropatias graves, endocrinopatias, hipertensão arterial crônica, doenças neurológicas, doenças autoimunes, alterações genéticas maternas, hanseníase, tuberculose, fatores relacionados a história reprodutiva anterior, como morte intrauterina ou perinatal, abortamento habitual, malformações fetais ou arritmia fetal, entre outros Ministério da saúde, (2012).

Os casos de fatores de risco que indicam encaminhamento à urgência/emergência obstétrica, são as que apresentam síndromes hemorrágicas, suspeita de pré-eclâmpsia, crise hipertensiva, amniorrexe prematura (perda de líquido vaginal), isoimunização $\mathrm{Rh}$, anemia grave, trabalho de parto prematuro, vômitos incoercíveis, restrição de crescimento intrauterino, IG a partir de 41 semanas confirmado, hipertermia, entre outros Martins et al., (2015).

O diagnóstico pré-natal objetiva a detecção das cardiopatias congênitas graves, onde o exame de imagem realizado pelas gestantes, o ultrassom morfológico deve ser feito cuidadosamente e a estrutura do coração bem analisada. Os principais achados para detecção de cardiopatias são: sopro cardíaco, cianose, taquipneia e arritmia cardíaca. Porém, muitas vezes apenas o ultrassom pode se tornar ineficaz nos grupos de baixo risco, fazendo com que não haja detecção dos defeitos cardíacos podendo resultar em óbitos fetais. Devido a isso, muitos sugerem que a ecocardiografia fetal deve ser realizada em todas as gestantes, pois ela é considerada técnica padrão ouro para diagnóstico cardíaco fetal e pode detectar mais precisamente todas as anomalias cardíacas Pedra et al., (2019); Santos; Menezes \& Sousa, (2013).

Algumas condições clínicas maternas aumentam os riscos para cardiopatias fetais, sendo consideradas fatores para indicação de ecocardiografia fetal para ser feito o rastreamento. Os fatores que contem risco maior que $2 \%$ são: diabetes melito materno pré-gestacional, diabetes melito materno diagnosticado no primeiro trimestre, fenilcetonúria materna, anticorpos 
materno anti-RO e anti-LA, ingestão materna de medicamentos (IECA, ácido retinóico, anti-inflamatórios não hormonais no terceiro trimestre), rubéola materna no primeiro trimestre, infecção materna com suspeita de miocardite fetal, gestação por reprodução assistida, cardiopatia congênita em parentes (mãe, pai ou irmão portador), herança mendeliana associada a cardiopatia congênita em parentes de primeiro grau ou segundo grau, suspeita de cardiopatia congênita pelo ultrassom obstétrico/morfológico, cariótipo fetal anormal, ritmo cardíaco fetal irregular, bradicardia ou taquicardia, gestação gemelar monocoriônica, hidropisia fetal ou derrames Pedra et al., (2019).

Os que correspondem ao risco entre 1 e $2 \%$ são: ingestão materna de medicações (anticonvulsivantes, lítio, vitamina A, inibidores seletivos da recaptação da serotonina - somente paroxetina, anti-inflamatórios não hormonais em primeiro e segundo trimestre), cardiopatia congênita em parente de segundo grau, anormalidade fetal do cordão umbilical ou da placenta, anomalia intra-abdominal fetal. E os riscos que são menores que 1\% são diabetes melito materno gestacional, ingestão materna de medicações (inibidores seletivos da recaptação da serotonina - todos exceto paroxetina, agonista da vitamina k- varfarina), infecção materna diferente da rubéola com apenas soro conversão e cardiopatia congênita isolada em algum parente distante Pinto; Westphal \& Abrahão, (2016).

Os testes de triagem fetal, ultrassonografia e ecocardiograma objetivam a visualização das câmaras do coração. A Sociedade Internacional de Ultrassonografia em Obstetrícia e Ginecologia (ISUOG) orienta a ultrassonografia nas gestações de baixo risco, onde poderão identificar fetos com riscos de síndromes genéticas e malformações congênitas, visto que apresentam alguns problemas já citados. E as alterações cardíacas devem ser detectadas por meio do ecocardiograma fetal, o equipamento permite uma boa detecção de imagens e a partir de 18 semanas, todas as estruturas cardíacas podem ser observadas. No entanto, entre 24 e 28 semanas são obtidas as melhores imagens, pois o coração está maior. A avaliação precoce do coração também pode ser realizada, pela via transvaginal ou trasabdominal (após a $14^{\mathrm{a}}$ semana), e geralmente é indicada para as gestantes que apresentaram alto risco de cardiopatia fetal na triagem do primeiro trimestre Brasil, (2017).

Quando as cardiopatias são detectadas, podemos elencar os passos de detecção especificamente na Figura 10. O acompanhamento deve ser feito a partir de ecocardiografias em intervalos de tempo que variam de 2 a 6 semanas, os detalhes anatômicos devem ser observados e acompanhados, a fim de evitar o agravamento da cardiopatia ao longo da gestação. Intervenções ainda na vida fetal são feitas nos casos de estenose aórtica crítica e atrésia pulmonar com septo íntegro e deve ser considerada em casos específicos, pois os casos que se beneficiam com esses procedimentos invasivos devem ser bem estudados, para determinar qual a maneira mais precisa para cada caso. Quando chega o momento do nascimento do bebê com cardiopatia fetal, o cardiologista fetal vai coordenar o nascimento juntamente com obstetra, neonatologista, intensivista, incluindo serviços de cardiologia especializada, como hemodinamicista, ritmologista e cirurgia. Tudo isso vai facilitar, pois dentro de um centro de referência especializado vai se ter todos os recursos necessários e profissionais especializados para o atendimento do neonato nas primeiras horas após o nascimento (Chegar, 2015). 


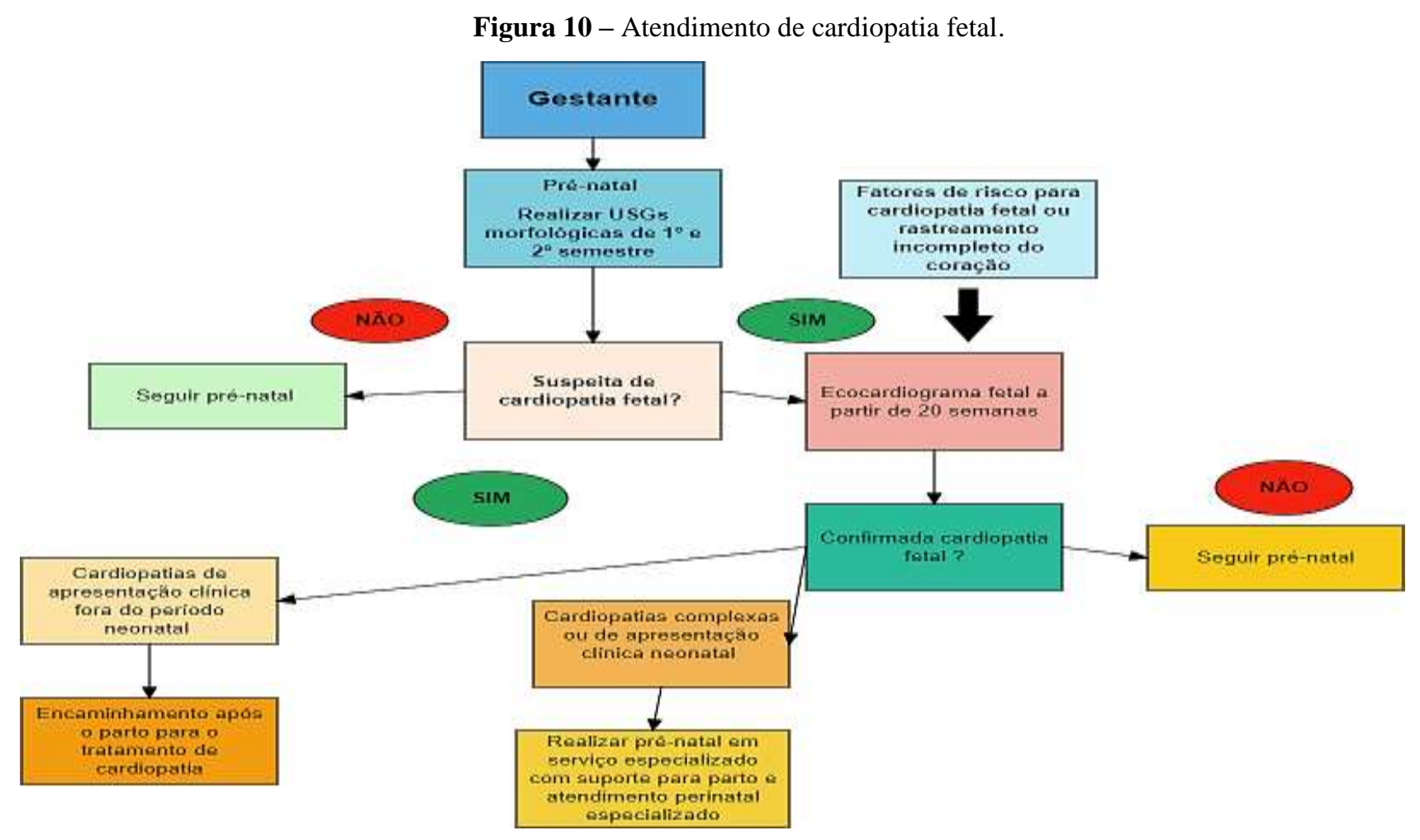

Fonte: Fluxograma Adaptado da Diretriz Brasileira de Cardiologia Fetal (2009).

O diagnóstico antenatal das cardiopatias fetais vai permitir o acompanhamento das patologias, com potencial de descompensacão hemodinâmica intraútero além disso vai permitir a programação dos casos nos quais as cardiopatias apresentarão repercussão hemodinâmica no período neonatal imediato. Com relação ao comportamento das alterações cardíacas na fase fetal é muito importante saber quais as alterações que podem evoluir com repercussão hemodinâmica no período intrauterino e quais necessitarão de condutas antes do nascimento, por administração de medicação ou por intervenções intrauterinas ou até mesmo antecipação do nascimento Silva et al., (2018).

As alteraç̃oes cardíacas no feto são classificadas em estruturais e funcionais. Com relação as estruturais, elas não apresentam comprometimento na fase intrauterina devido a fisiologia da circulação fetal. Sua repercussão clínica vai acontecer logo após o nascimento, onde vai ocorrer o fechamento fisiológico dos shunts intracardíacos. Os corações anatomicamente normais também poderão apresentar algum comprometimento em sua atividade, ou por acometimento do miocárdio, alterações ritmo ou débito cardíaco ou modificações do fluxo nos shunts intracardíacos. São essas condições que podem apresentar repercussão hemodinâmica no feto, indicando a necessidade de tratamento antes do nascimento Pedra et al., (2019).

Vale ressaltar a importância do acompanhamento multidisciplinar dos fetos com cardiopatias, visto que apresentam maior probabilidade de os mesmos apresentarem associação com síndromes genéticas ou malformações extracardíacas graves, o que eleva a mortalidade pós natal Silva et al., (2018).

Apesar de todos os avanços no diagnóstico pré-natal, na ecografia obstétrica e na ecocardiografia fetal, um grande número de recém-nascidos com cardiopatias congênitas nasce sem o diagnostico definido. E devido aos altos custos dos exames, por necessitarem de profissionais especializados para a realização dos mesmos, torna-se inviável que todos os neonatos recebam os procedimentos citados anteriormente Aguiar et al., (2018).

Diante disso, após o nascimento, estão sendo incorporadas na prática hospitalar algumas ações para a detecção de patologias associadas ao sistema cardiovascular, dentre elas está a oximetria de pulso, conhecida como teste do coraçãozinho. 
O teste vai desempenhar um papel muito importante na detecção dos neonatos, para que se tenha o conhecimento da doença e possa realizar os procedimentos adequados antes dos mesmos receberem a alta hospitalar Aguiar et al., (2018).

O teste do coraçãozinho é um método de triagem seguro, efetivo, de baixo custo, além de ser um método indolor, não invasivo, benéfico, de fácil manuseio e aplicável na assistência ao recém-nascido. Fazendo associação do mesmo ao exame físico, que deve observar presença de cianose central ou periférica, palpação do precário e pulsos periféricos, presença de insuficiência cardíaca e ausculta cardíaca para identificação de sopros, o procedimento se torna bastante eficaz no reconhecimento das cardiopatias nos primeiros dias de vida dos neonatos Queiroz \& Lucena, (2020).

Esses testes serão muito importantes para se fazer o encaminhamento e para a tomada de decisões dos procedimentos adequados a cada caso e qual a melhor conduta a ser seguida, para se iniciar a investigação da cardiopatia e o início do tratamento no momento certo a fim de aumentar sua eficácia Silva et al., (2018).

\section{Tratamento de Pacientes Prematuros com Doenças Cardíacas}

A cirurgia cardíaca é a principal forma de abordagem precoce com a finalidade de evitar óbitos. Apesar dos grandes avanços na cirurgia cardíaca crônica, nas técnicas de circulação extracorpórea e melhoras na habilidade de manejo pré e pósoperatório, os resultados promissores foram o declínio na mortalidade geral em todas as faixas etárias. Entretanto, a cirurgia cardíaca neonatal ainda apresenta taxas de mortalidade elevadas Krishnamurthy; Ratner\& Bacha, (2013).

Um em cada 10 neonatos não sobrevivem até a alta hospitalar após a cirurgia cardíaca. As lesões que necessitam de cirurgias cardíacas, na maioria das vezes são bastante complexas, requerem muitas habilidades técnicas dos médicos e vários anos de experiência para o domínio do procedimento, pois os neonatos apresentam questões desafiadoras com relação à estrutura, à canulação e à circulação extracorpórea. Axelrod; Chock\& Reddy, (2016).

Antes, os cirurgiões cardiovasculares pediátricos não tinham capacidade para reparar defeitos cardíacos simples em crianças, mas à medida que foram produzidos instrumentos mais modernos com tamanhos apropriados, as técnicas cirúrgicas foram aperfeiçoadas e os cirurgiões começaram a considerá-las. Por se tratarem de cirurgias complexas em corações minúsculos, a circulação pré-operatória anormal e os efeitos da circulação extracorpórea em órgãos imaturos são alguns fatores adicionais que colocam os neonatos em um maior risco de morte após a cirurgia Krishnamurthy; Ratner\& Bacha,(2013).

Embora hoje em dia haja abordagem cirúrgica para a maioria dos defeitos cardíacos congênitos, o debate continua em relação ao momento apropriado da intervenção cirúrgica para os neonatos prematuros com cardiopatias congênitas Axelrod; Chock \& Reddy (2016). O momento da cirurgia é levado em consideração a vários fatores pelo cirurgião cardiologista, os quais incluem o peso, a estabilidade clínica, o tipo de lesão cardíaca, a complexidade e o tipo de cirurgia. O procedimento específico vai depender da idade e do tamanho do bebê, e normalmente a cirurgia é adiada na primeira semana de vida, para permitir a avaliação do estado clínico do bebê e é liberada quando está fisiologicamente estável e quando as morbidades não cardíacas estão melhorando Lynema; Fifer\& Laventhal, (2016).

$\mathrm{O}$ atendimento às crianças com cardiopatias congênitas no Brasil é um dos grandes desafios do SUS, pois no país a distribuição geográfica dos centros de referências de cardiologia e cirurgia cardíaca pediátrica são determinantes para os índices de mortalidade. No Brasil, existem apenas 69 centros de cirurgia cardíaca pediátrica, e no presente estudo "Mortalidade para Cardiopatias Congênitas e Fatores de Risco Associados em Recém-Nascidos. Um Estudo de Coorte, evidencia que o tempo médio de permanência dos recém-nascidos com cardiopatias congênitas no hospital foi de 75 dias e $25 \%$ dos recémnascidos já haviam morrido no período neonatal Lopes et al., (2018).

No ano de 2017, o Ministério da Saúde criou um projeto federal para ampliar a assistência às crianças com cardiopatias congênitas, com o principal objetivo de aumentar em 30\% os atendimentos por ano, totalizando cerca de 12.600 procedimentos, o que iria impactar na redução da mortalidade. Porém, o que se faz necessário em nosso país são intervenções, 
remodelagem e reestruturação nas diversas fases do seu processo, com a finalidade de atingir as metas. Pois, além do investimento financeiro, envolverá questão política, social e cultural específica de cada região Lopes et al., (2018).

Com relação ao tratamento medicamentoso, em um estudo realizado no ano de 2011, onde 75 pacientes que apresentavam cardiopatias congênitas do tipo persistência do canal arterial, desses, 57 receberam medicação e 34 foram medicados com ibuprofeno. As drogas indometacina e ibuprofeno são bastante utilizadas para o fechamento farmacológico do canal arterial, onde entre elas, a principal escolha ainda é o ibuprofeno decorrente dos menores índices de efeitos colaterais. Outros fármacos como os diuréticos também são utilizados, com a principal finalidade de controlar os sinais e os sintomas pulmonares e periféricos da congestão, e fazer a retenção de água e sódio. A droga de primeira escolha dos diuréticos é a furosemida, devido a sua ação, mesmo quando há insuficiência renal. Existem outros fármacos que são: aldactone, espironolactona e hidroclotiazida, que são utilizados para o tratamento de hipertensão Santos; Menezes\& Sousa, (2013).

Outras drogas também utilizadas por cardiopatas são as drogas vasoativas: dopamina, dobutamina e adrenalina, usadas para manter a pressão arterial. Propanolol, com a finalidade de reparar arritmias, hipertensão e prevenção da enxaqueca. Digitálicos, a digoxina tem a função de agir na frequência cardíaca, corrigindo arritmias. Amiodarona, que é um antirrítimico que age nas arritmias ventriculares. E a atropina, utilizada para tratar bradiarritmias, com bloqueio atrioventricular Santos; Menezes \& Sousa, (2013).

As prostaglandinas E1 (PGE1) também são utilizadas para melhorar a estabilização clínica dos recém-nascidos. Ela tem ação dilatadora no tecido do canal arterial até o final da $4^{\text {a }}$ semana de vida, após esse período sua resposta é insatisfatória, pois ela tem resposta apenas sobre o canal arterial, e passado esse período o canal vai se fechar e não vai poder exercer sua ação dilatadora. As PGE1 podem ser utilizadas nas seguintes cardiopatias: cardiopatias com fluxo pulmonar dependente do canal arterial, ex.: atresia pulmonar e atrésia aórtica e nas cardiopatias com circulação em paralelo, ex.: transposição das grandes artérias Brasil. Ministério da saúde.,(2014).

\section{Acompanhamento e Estimulação Precoce do Bebê Prematuro com Cardiopatia}

A estimulação precoce é o termo que envolve uma variedade de estímulos que tem por objetivo o desenvolvimento cognitivo de lactantes e crianças e pode ser definido como um programa de acompanhamento e tratamento multifuncional para os recém-nascidos que apresentam riscos ou alguma deficiência. A estimulação precoce prioriza o atendimento de crianças de zero a três anos de idade e envolve terapias tradicionais com fisioterapia, terapia ocupacional e fonoaudiologia. O tratamento é benéfico para os recém-nascidos que apresentam condições ou agravos de saúde que venham a interferir no desenvolvimento neuropsicomotor como a prematuridade, paralisia cerebral, doenças congênitas, entre outras (Iff/Fiocruz).

Os pacientes com pouca idade que são submetidos a cirurgia cardíaca têm um maior risco de comprometimento no desenvolvimento, que podem repercutir ao longo da vida. Os mesmos podem apresentar atraso cognitivo e motor pós cirurgia cardíaca durante a primeira infância, e trará consequências durante a fase final da infância e até na vida adulta Leal et al., (2016).

O desenvolvimento motor é resultado de interações entre potenciais biológicos geneticamente determinados e circunstâncias ambientais. Portanto, é influenciável pela interação entre o indivíduo e o contexto no qual ele está inserido. As principais causas motoras são: baixo peso ao nascer, alterações respiratórias e neurológicas, infecções neonatais, desnutrição, baixas condições socioeconômicas, prematuridade e distúrbios cardiovasculares Leal et al., (2016).

Portanto, evidencia-se que se faz necessário a inserção dos prematuros com cardiopatias congênitas em programas de vigência para avaliação desses riscos e intervenção precoce. Visto que, os mesmos necessitam de um tratamento diferenciado e têm longas internações hospitalares, se fazendo necessário identificar se além das limitações da doença, podem desenvolver problemas motores relacionados Leal et al., (2016). 
No presente estudo, com o tema "Avaliação do Desenvolvimento Motor de Crianças Portadoras de Cardiopatia Congênita - 2016", a análise concluiu que as crianças com cardiopatias congênitas apresentavam atraso no desenvolvimento motor, onde os estudados apresentavam idades motoras inferiores a esperada, onde pode-se relacionar aos fatores fisiológicos, igualmente aos encontrados nas cardiopatias cianogênicas, que a oxigenação do cérebro faz com que ele fique menos eficiente.

A conduta necessária da consequência das cardiopatias deve ser tomada com cautela, pois vai interferir diretamente na qualidade de vida da criança. O terapeuta ocupacional pode atuar orientando e minimizando o atraso no desenvolvimento motor. E o fisioterapeuta pode utilizar as técnicas de estimulação precoce, onde vai informar quais as atividades motoras ideal para criança com base na sua idade cronológica, por meio de manuseios e facilitação de postura e movimento Iff/fiocruz; Leal et al., (2016).

É imprescindível a orientação aos pais, para que os mesmos também estimulem e torne favorável o ambiente da criança e o desenvolvimento de suas habilidades. Fazendo assim com que todos trabalhem em prol de minimizar as consequências das próximas etapas de desenvolvimento, para a aprendizagem, socialização e a vida adulta.

\section{Correlação entre a Biomedicina e Doenças Cardíacas}

As doenças cardiovasculares congênitas, na porcentagem de 25 a 30\% estão associadas a anomalias extra cardíacas, e alguns defeitos são associados com síndromes genéticas. As aneuploidias são as mais identificadas causas genéticas associadas, e com relação as anormalidades citogenéticas varia de 9\% a 18\% Saliba et al., (2020).

O desenvolvimento do coração é bastante complexo, vários fatores moleculares e epigenéticos estão envolvidos. O conhecimento das alterações genéticas subjacentes as cardiopatias pode ser um fator determinante no prognóstico e na antecipação prévia do tratamento de complicações que possam vir a acontecer, como resposta inflamatória sistêmica, arritmias e insuficiência cardíaca precoce Saliba et al., (2020).

Segundo o Conselho Regional de Biomedicina $3^{\circ}$ região CRBM3, (2019), o biomédico geneticista pode contribuir na investigação das doenças cardíacas, visto que envolve fatores genéticos e o mesmo atua na identificação de doenças e modificações genéticas, através da realização de análises bioquímicas de enzimas, substratos, e produtos de diversas vias metabólicas. O diagnóstico de distúrbios metabólicos identificados precocemente fornece ao médico mais tempo de prevenir sequelas e em alguns casos a morte.

O profissional geneticista também realiza sequenciamento de DNA, faz a análise dos fragmentos e desenvolve estratégias de investigação e interpretação dos dados obtidos e o acompanhamento. O mesmo, também pode ser bastante eficaz no acompanhamento genético, que na maioria das vezes são solicitados durante o pré- natal ou pós-natal, quando existem riscos para o desenvolvimento de alguma alteração genética, seja pelo histórico familiar ou background étnico, crianças com alterações genéticas já diagnosticadas, gravidez com idade materna maior que 35 anos, abortamentos repetitivos, infertilidade conjugal, consanguinidade CRBM3, (2019).

O processo de acompanhamento genético envolve a interpretação dos históricos médico e familiar para se analisar a estimativa de ocorrência ou recorrência de doença genética, educação sobre herança familiar, testes, prevenção, recursos disponíveis e pesquisa, aconselhamento para promover escolhas previamente informadas e adaptação aos riscos e auxílio aos pais/ pacientes a compreenderem e tomar as decisões do que pode ser feito CRBM3, (2019).

Com relação à cirurgia cardíaca, o biomédico pode atuar diretamente por meio da perfusão e circulação extracorpórea. A cirurgia com circulação extracorpórea foi uma grande conquista na área médica e na área biológica do século XX, possibilitou a cura de diversas doenças cardíacas. Por meio dela é possível corrigir os defeitos do coração, pois possibilita adentrar as cavidades cardíacas e corrigir os defeitos cardíacos congênitos ou adquiridos. Durante esse período, em que a circulação sanguínea e a respiração são mantidas por meios artificiais, a fisiologia deve ser monitorada dentro dos parâmetros 
de normalidade CRBM5, (2020).

A circulação extracorpórea é realizada por meio de um equipamento que substitui as funções exercidas pelo coração, rins e pulmões. Esse procedimento é utilizado em cirurgias cardíacas, retiradas de tumores e insuficiência pulmonar. Cabe ao biomédico com habilitação em perfusão, montar o dispositivo que drena, filtra, oxigena e re-injeta o sangue no paciente, assim como, também realizar a monitorização dos indicadores, como pressão, temperatura, coagulação e fluxo sanguíneo Braile, (2010).

Nas crianças de muito baixo peso, a circulação permite restaurar lesões em cardiopatias congênitas complexas, permite ao cirurgião o acesso às áreas de difícil acesso e fazer as correções necessárias. A cirurgia cardíaca com circulação extracorpórea é bastante complexa, o profissional deve ter conhecimentos profundos e raciocínio rápido para a tomada de decisões automáticas e imediatas Braile, (2010).

Com relação aos riscos maternos, já citados e que podem desencadear cardiopatias congênitas, como diabetes melito materno, fenilcetonúria materna, anticorpos maternos anti-RO e anti-LA e rubéola, todas as alterações são diagnosticadas por exames laboratoriais, onde o biomédico vai contribuir para a detecção Regional, (2018).

A diabetes mellitus é diagnosticada por meio do teste de glicose em jejum ou do teste oral de tolerância a glicose, onde vai depender da indicação do médico. A fenilcetonúria que é uma condição hereditária, é detectada previamente no nascimento por meio do teste do pezinho e vai persistir por toda a vida, pois é uma doença hereditária que não tem cura, só vai necessitar de uma dieta adequada. Anti- RO e anti-LA são os anticorpos das doenças reumáticas, lúpus eritematoso e síndrome de Sjogren, o diagnóstico é realizado por meio de testes sorológicos. Já a rubéola também é detectada por meio de testes sorológicos para a detecção de anticorpos IgM específicos da rubéola Ministério da saúde, (2019).

Diversos outros exames laboratoriais são solicitados durante o pré-natal, onde podem ser detectadas várias doenças que também comprometem a gestação. O papel do biomédico é de grande valia, visto que, como já mencionado o mesmo pode atuar diretamente na detecção de diversas patologias por meio dos exames laboratoriais. E como citado, nas cardiopatias congênitas, ele também tem sua contribuição profissional. Ministério da saúde, (2019); Regional, (2018).

\section{Conclusão}

A Biomedicina é parte da ciência que trata de diversos assuntos, está voltada a área da pesquisa de novas doenças, prevenção, exames e objetiva o desenvolvimento de novos métodos para aumento da qualidade de vida. $\mathrm{O}$ assunto estudado nesse trabalho tem grande importância, visto que é pouco abordado e exige, a partir do momento da detecção do problema coronariano, uma equipe multiprofissional, onde pode está incluído o biomédico.

No presente estudo realizado por Costello et al., (2010), que tem como título "O nascimento antes de 39 semanas de gestação", teve por objetivo determinar se existia uma associação entre idade gestacional no parto e os resultados adversos em neonatos com doença cardíaca congênita. Foi realizado um estudo de coorte com 971 neonatos no período de 2002 a 2008 e os resultados foram que os neonatos que nasceram antes de 39 semanas têm risco de mortalidade aumentada. Não foi evidenciando o momento ideal do parto, mas fica a ressalva que os cardiologistas devem investigar cuidadosamente o momento ideal para o nascimento.

Em cuidados cardíacos neonatais, uma perspectiva Krishnamurthy; Ratner\&Bacha, (2013), torna evidente que os bebês nascidos antes do período gestacional correm substancialmente mais riscos e enfrentam diversos desafios. Esse trabalho não especifica o momento ideal da cirurgia cardíaca, mas exemplifica que as cirurgias cardíacas com circulação extracorpórea têm sido realizadas com sucesso em pacientes prematuros. Deixa claro que os recém nascidos prematuros com doenças cardíacas requerem maiores cuidados da equipe profissional treinada e experiente.

Na pesquisa intitulada "Caracterizando o perfil dos recém nascidos com cardiopatia congênita em uma maternidade de 
alto risco em Aracaju" Santos; Menezes\& Sousa, (2013), foi realizado estudo transversal, retrospectivo com abordagem quantitativa e análise descritiva de 168 recém nascidos com cardiopatias congênitas. Foram descritas as cardiopatias, achados dos sintomas mais frequentes, sendo eles: cianose, sopro, dispneia. Tornando também evidente que $67 \%$ nasceram com idade gestacional menor que 34 semanas, onde foram encontrados 24 tipos de cardiopatias, entre elas as mais evidentes foram: persistência do canal arterial, comunicação interventricular e interatrial. O tratamento medicamentoso foi o mais instituído, $75 \%$ receberam alta e $23 \%$ tiveram óbito.

No estudo sobre mortalidade para cardiopatias congênitas e fatores de risco associados em recém-nascidos - um estudo de coorte Lopes et al., (2018), evidencia os fatores de risco nos óbitos dos recém nascidos com cardiopatia congênita crítica ou complexa no período neonatal, os resultados mostraram que os nascidos prematuros, baixo peso, tem taxas de mortalidade maiores e além disso, apresentam uma gama de outras patologias que pode fazer com que aumente mais ainda a gravidade do seu problema. O artigo também chama atenção para os desafios que são enfrentados no tratamento das mesmas, evidenciando que a alta taxa de letalidade requer atenção crítica para os mesmos e uma reestruturação da rede de assistência especializada.

Dos vários autores estudados e com todas as análises a respeito de doenças cardíacas, as principais respostas a respeito do desenvolvimento das mesmas foi que o fato dos bebês nascerem prematuros já é um preditor de fator de risco, visto que, o coração não atingiu a maturidade suficiente e ainda não está preparado para a vida extrauterina, podendo vir a desenvolver cardiopatias.

Além disso, os fatores genéticos e os hábitos adotados pela gestante no decorrer da gravidez contribuem significativamente para a saúde do bebê. Fazendo- se necessário o acompanhamento pré-natal para rastreio e caso seja detectada alguma suspeita de risco para futuras cardiopatias congênitas, as medidas corretas deverão ser adotadas imediatamente.

Apesar do tratamento medicamentoso, a maioria das cardiopatias detectadas nos prematuros ainda são tratadas com procedimentos cirúrgicos, e apesar dos avanços nas técnicas das cirurgias, o fato da realização nos prematuros ainda é bastante preocupante, pois é uma cirurgia de alta complexidade e que exige bastante conhecimento e experiência dos profissionais envolvidos.

Portanto, a partir dos resultados encontrados, ainda existem poucos trabalhos científicos a respeito do tema, fazendose necessário mais estudos, pois é um tema de bastante relevância e que pode contribuir significativamente para a área da saúde pública, bem como para os profissionais da área da Biomedicina que podem atuar conjuntamente com as equipes médicas que tratam de problemas coronarianos.

\section{Referências}

Aguiar, C. B. et al. (2018) Teste do coraçãozinho: importância da oximetria de pulso em neonatos para detecção precoce de cardiopatias. Revista Eletrônica Acervo Saúde, v. Esp., n. 12, p. S1349-S1357.

Axelrod, D. M.; Chock, V. Y.; Reddy, V. M. (2016) Management of the Preterm Infant with Congenital Heart Disease. Clinics in Perinatology, v. 43, n. 1, p. 157-171. http://dx.doi.org/10.1016/j.clp.2015.11.011.

Bailliard, F.; Anderson, R. H. Tetralogy of Fallot. (2009). Orphanet Journal of Rare Diseases,v.4,n.1.

Balsells, M. M. D. et al. (2018). Avaliação do processo na assistência pré-natal de gestantes com risco habitual. Acta Paulista de Enfermagem, v. 31, n. 3, p. $247-254$.

Biomédico geneticista apresenta detalhe sobre aconselhamento genético (AG).(2019).Conselho Regional de Biomedicina $3^{\circ}$ região.

Braile, D. M. (2010). Circulação Extracorpórea. Revista Brasileira de Cirurgia Cardiovascular,v. 25, n. 4, p. III-V.

Brasil. ministério da saúde. (2015). Atenção à Saúde do Recém-Nascido. Cuidados Gerais. [s.l: s.n.]. v. 3 
Brasil. ministério DA SAÚDE. (2012). Secretaria de Atenção à Saúde. Departamento de Atenção Básica. Atenção ao pré-natal de baixo risco / Ministério da Saúde. Secretaria de Atenção à Saúde. Departamento de Atenção Básica. - Brasília : Editora do Ministério da Saúde. 318 p.: il. - (Série A.

Brasil. (2020) Diagnóstico precoce de cardiopatias congênitas. [s.l: s.n.].

Chegar, O. P. (2015) Cardiopatias Congênitas , um novo olhar: diagnóstico e tratamento. Revista Da Sociedade De Cardiologia Do Estado De São Paulo, v. 25, n. 3 , p. 60.

Coarctação de aorta. (2020) Dr Bruno Rocha cirurgia cardiovascular. http://brunorocha.com.br/coarctacao-de-aorta.

Comunicação interatrial. (2020) Dr Bruno Rocha cirurgia cardiovascular.

Comunicação interventricular. (2018). Hospital Infantil Sabará.

Cortellazzi, T.; Ecocardiograma, N. (2017) Estenose aórtica subvalvar. http://sociedades.cardiol.br/pi/pdf/jornal/dezembro-2017.pdf.

Costello, J. M. et al. (2010) Birth before 39 weeks' gestation is associated with worse outcomes in neonates with heart disease. Pediatrics, v. 126 , n. 2.

Dadvand, P. et al. (2009) Descriptive epidemiology of congenital heart disease in Northern England. Paediatric and Perinatal Epidemiology, v. 23, n. 1, p. 5865 .

Silva S, A. D.; Menezes \& G. De A. De Sousa, D. S. (2013)Perfil dos Recém- Nascidos com Cardiopatia Congênita em uma Maternidade de Alto Risco do Município de Aracaju. Caderno de Graduação - Ciências Biológicas e da Saúde - UNIT - SERGIPE, v. 1, n. 3, p. 59-70

Estenose Aórtica. (2020) Hemodinâmica e Cardiovascular Invasiva - Hci Med.

Higino, A. D. et al. (2015). Pravalência de fatores de risco para doenças cardiovasculares em crianças e adolescentes do Núcleo de Amparo ao Menor. Revista Científica da Escola de Saúde da Universidade Potiguar, v. 1, n. 4, p. 59-67.

Intervcenter realiza tratamento de cardiopatia congênita em válvula de recém-nascido. (2020). Coluna Cleber Toledo.

Krishnamurthy, G.; Ratner, V. \& Bacha, E. (2013). Neonatal cardiac care, a perspective. Seminars in Thoracic and Cardiovascular Surgery: Pediatric Cardiac Surgery Annual, v. 16, n. 1, p. 21-31.

Leal, L. S. et al. (2016) Avaliação do Desenvolvimento Motor de Crianças Portadoras de Cardiopatia Congênita. v. 29, n. 2, p. $103-109$.

Lopes, A. A.; Mesquita, S. M. F. C (2014) Does Repair Always Mean Cure? Arquivos Brasileiros de Cardiologia, v. 103, n. 6, p. $446-448$.

Lopes, S. A. V. D. A. et al (2018). Mortality for critical congenital heart diseases and associated risk factors in newborns. A cohort study. Arquivos Brasileiros de Cardiologia, v. 111, n. 5, p. 666-673, 2018.

Lynema, S.; Fifer, C. G.; Laventhal, N. T. (2016). Perinatal Decision Making for Preterm Infants with Congenital Heart Disease: Determinable Risk Factors for Mortality. Pediatric Cardiology, v. 37, n. 5, p. 938-945. https://link.springer.com/article/10.1007/s00246-016-1374-y.

Mangili, G.; Garzoli, E.; Sadou, Y. (2018). Feeding dysfunctions and failure to thrive in neonates with congenital heart diseases. Pediatria Medica e Chirurgica,v.40,n.1,p.1-4.

Martins, A. C. M. et al. (2015). Protocolos de encaminhamento para Obstetrícia (Pré-Natal de Alto Risco).

Moons, P. et al. (2009). Congenital heart disease in 111225 births in Belgium: Birth prevalence, treatment and survival in the 21st century. Acta Paediatrica, International Journal of Paediatrics, v. 98, n. 3, p. 472-477.

Morais, S.; Mimoso, G. (2013). Oximetria de pulso no diagnóstico de cardiopatia congénita. Sugestões para a implementação de uma estratégia de rastreio. Acta Pediátrica Portuguesa, v. 44, n. 6, p. 343-347.

Perfusão e circulação extracorpórea: suporte de vida fora do corpo. (2020). Conselho Regional de Biomedicina $5^{\circ}$ região.

Pedra, S. R. F. F. et al. (2019) Brazilian fetal cardiology guidelines. Arquivos Brasileiros de Cardiologia, v. 112, n. 5, p. 600-648.

Pereira Pinto, C.; Westphal, F.\& Abrahão, A. R. (2018). Fatores de riscos materno associados à cardiopatia congênita Maternal risk factors associated with congenital heart disease. J Health Sci Inst, v. 36, n. 1, p. 34-42, 2018.

Persistência do canal arterial.(2011) Prematuridade.

Queiroz, I. M. A.; Lucena, G. P. DE. A importância do teste do coraçãozinho no diagnóstico precoce de cardiopatias congênitas. Revista Recien - Revista Científica de Enfermagem, v. 10, n. 29, p. 145-154, 2020.

Regional, C. Conselho Regional De Biomedicina 1a. n. 11, p. 10927, 2018

Saliba, A. et al. Genetic and genomics in congenital heart disease: a clinical review. Jornal de Pediatria (Versão em Português), v. 96, n. 3, p. 279-288, 2020.

Secretaria de estado da saúde do rio grande do sul. Guia do pré-natal na atenção básica. p.15, 2018.

Silva, L. D. C. et al. Diagnóstico precoce das cardiopatias congênitas: Uma revisão integrativa. JMPHC | Journal of Management \& Primary Health Care | ISSN 2179- 6750, v. 9, p. 1-24, 2018. Disponível em: https://www.jmphc.com.br/jmphc/article/view/336/731. Acesso em: 22 out. 2020. 
Research, Society and Development, v. 10, n. 7, e50710716917, 2021

(CC BY 4.0) | ISSN 2525-3409 | DOI: http://dx.doi.org/10.33448/rsd-v10i7.16917

Sociedade brasileira de pediatria (SBP). Diagnóstico precoce de cardiopatia congenital crítica: oximetria de pulso como ferramenta da triagem neonatal.n.5,p.5,2011.

Tadashi ywata de carvalho, A. et al. Tratamento endovascular da coarctação da aorta: relato de caso. Jornal Vascular Brasileiro, v. 11, n. 1, p. 57-61, 2012.

Tetralogia de fallot. Pediatria ufcspa,[200?]. https://pediatriaufcspa.wixsite.com/pediatria/tetralogia-de-fallot.

Valdes, A. Embriologia Humana. [s.l: s.n.]. v. 53. https://uab.ufsc.br/biologia/files/2020/08/Embriologia-Humana.pdf. 